\title{
Diseño e implementación de una secuencia didáctica para la enseñanza del concepto de elemento químico en educación secundaria
}

Dulce María López-Valentín ${ }^{1}$

Universidad Pedagógica Nacional, México

Autor de correspondencia: 1dvalentin@upn.mx Recibido: 06 de junio de 2020 Revisado: 30 julio de 2020 Aprobado: 17 de octubre de 2020 Publicado: 20 de noviembre de 2020

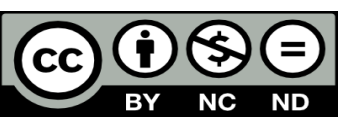

\section{Resumen}

Este estudio propone la implementación de una secuencia didáctica basada en el modelo de aprendizaje como investigación orientada, para la enseñanza del concepto de elemento químico en la educación secundaria. Su diseño se ha basado en las ideas previas de los estudiantes y en el análisis histórico sobre este concepto. La evaluación se ha realizado al comparar grupos de estudiantes que han seguido esta secuencia didáctica con grupos de estudiantes que han mantenido una enseñanza tradicional. Los resultados revelan que los estudiantes de los grupos experimentales respecto a los grupos control: 1) presentan un mejor manejo en la definición procedimental de sustancia en el nivel macroscópico; 2) definen mejor el concepto de elemento químico en el marco de la teoría daltoniana; 3) distinguen mejor los conceptos de sustancia simple y elemento químico; y 4) presentan una mejor distinción del concepto microscópico de mezcla - de sustancias simples o de sustancias compuestascuando es utilizada una secuencia didáctica de orientación constructivista.

Palabras claves: elemento químico, primer nivel de educación secundaria, enseñanza de las ciencias, enseñanza de la química

Para citar este artículo: López, D. (2020). Diseño e implementación de una secuencia didáctica para la enseñanza del concepto de elemento químico. Praxis \& Saber, 11(27), e11116. https://doi. org/10.19053/22160159.v11.n27.2020.11116 


\title{
Design and implementation of a teaching-learning sequence of the chemical element concept in high school education
}

\begin{abstract}
This article proposes the implementation of a teaching-learning sequence based on the model of learning in the context of an oriented research, in order to improve the teaching of the chemical element concept in high school education. Its design is based on the students' existing ideas and on the historical analysis about this concept. The evaluation was made by comparing groups of students who followed this didactic sequence to groups of students who maintained a traditional teaching. The results show that the students in the experimental groups compared to the control groups: 1) they present a better understanding of the procedural definition of substance at the macroscopic level; 2) they better define the concept of chemical element within the framework of the Daltonian theory; 3) they better distinguish the concepts of simple substance and chemical element; and 4) they demonstrate a better differentiation of the microscopic concept of mixture-of simple substances or of compounds-when a didactic sequence of constructivist orientation is used.
\end{abstract}

Keywords: chemical element, junior high school, science teaching, chemistry teaching

\section{Desenho e implementação de uma sequência didática para a instrução do conceito de elemento químico no ensino secundário}

\section{Resumo}

Este estudo propõe a implementação de uma sequência didática baseada no modelo de aprendizagem como investigação orientada, para a instrução do conceito de elemento químico no ensino secundário. Seu desenho baseou-se nas ideias anteriores dos estudantes e na análise histórica deste conceito. A avaliação foi feita comparando grupos de estudantes que seguiram esta sequência didática com grupos de estudantes que mantiveram o ensino tradicional. Os resultados revelam que os estudantes dos grupos experimentais em relação aos grupos de controle: 1) apresentam um melhor manejo na definição processual de substância no nível macroscópico; 2) definem melhor o conceito de elemento químico no âmbito da teoria daltônica; 3) distinguem melhor os conceitos de substância simples e elemento químico; e 4) apresentam uma melhor distinção do conceito microscópico de mistura - de substâncias simples ou substâncias compostas - quando é utilizada uma sequência didática de orientação construtivista.

Palavras-chave: elemento químico, primeiro nível de ensino secundário, ensino de ciências, ensino de química 


\section{Objetivo}

El objetivo de este trabajo es presentar el diseño y desarrollo de una secuencia didáctica sobre el concepto de elemento químico para estudiantes de educación secundaria al aplicar el modelo de enseñanza-aprendizaje como investigación orientada.

\section{Metodología}

El concepto de elemento químico [CEQ] es considerado como un prerrequisito para el estudio de la química, imprescindible para poder entender el cambio químico, tanto en el nivel macroscópico como en el submicroscópico. Al introducirse en la teoría atómica de Dalton, el CEQ explica y unifica todos los conceptos macroscópicos conocidos al principio del siglo XIX, ya que ofrece explicaciones submicroscópicas para los mismos: 1) la diferencia entre sustancia y mezcla; 2) la diferencia de comportamiento entre las sustancias simples y los compuestos; y 3) la diferencia entre un proceso físico y un proceso químico. En este último caso, cabe especificar que la idea de elemento permite predecir qué sustancias se pueden formar en un cambio químico y cuáles no, puesto que en una reacción química las sustancias no permanecen, pero los elementos químicos sí se mantienen.

Por otro lado, el CEQ también permite comprender otros conceptos subsecuentes y más complejos, como lo son la cantidad de sustancia y la estequiometría de las reacciones químicas (López-Valentín \& Furió-Más, 2013).

Es un concepto estructurante de la química porque:

- Permite explicar la diversidad de materiales ordinarios existentes con unos pocos elementos químicos, constituyendo este conjunto una estructura unitaria, y

- La búsqueda de esta estructura unitaria también posibilita la explicación de los cambios materiales que suceden en las reacciones químicas. Es una estructura modélica que supone la conservación o permanencia de aquellos elementos en las transformaciones químicas de los materiales (López-Valentín, 2008, p. 21).

Con respecto al aprendizaje particular del CEQ, hay una serie de consideraciones que sería conveniente tener en cuenta - ideas previas, obstáculos epistemológicos, etc. - que se dan frecuentemente en los estudiantes de educación secundaria. Estas dificultades pueden ser consecuencia del uso de una didáctica ajena a la metodología científica por el profesorado y al desconocimiento de la evolución histórica de los conceptos (Caamaño et al., 1983; Furió-Más \& Domínguez-Sales, 2007; Furió-Más et al., 2012; Laugier \& Dumon, 2003; López-Valentín, 2008).

\section{Diseño de la secuencia didáctica}

Para el diseño de la secuencia didáctica, se tuvieron en cuenta los siguientes aspectos:

a) Las dificultades que presentan los estudiantes respecto a la comprensión del CEQ.

b) El análisis histórico del CEQ, específicamente su evolución en los diferentes modelos históricos hasta los actuales.

c) La utilización del modelo de aprendizaje como investigación orientada para secuenciar las actividades propuestas.

Según los puntos anteriores, se elaboró una secuencia didáctica cuya consigna fuese 
superar las dificultades de aprendizaje detectadas por la investigación respecto al CEQ. Por ello, esta contribución pretende contestar las siguientes preguntas de investigación:

1. ¿Cómo diseñar una secuencia didáctica que tenga en cuenta los aspectos mencionados para mejorar el aprendizaje del CEQ en estudiantes de secundaria?

2. ¿En qué medida la aplicación de la secuencia didáctica puede favorecer la superación de las dificultades de los estudiantes respecto al CEQ?

Para responder estas preguntas empezaremos por revisar cada uno de los aspectos mencionados:

\section{Dificultades que presentan los estudiantes respecto a la comprensión del CEQ}

La investigación en didáctica ha detectado las siguientes dificultades en los estudiantes (Lopez-Valentín \& Furió-Más, 2013, p. 1998):

No entienden el concepto macroscópico de sustancia y lo confunden con el concepto de material - mezcla de sustancias-. En general, no utilizan la definición macroscópica de sustancia como "material puro, no mezclado, que posee un conjunto de propiedades características y constantes”. Esto también se puso de manifiesto en Furió-Más y DomínguezSales (2007).

No comprenden el concepto de elemento químico. En general, no saben definirlo, pero, cuando lo hacen, tienden a superponer las definiciones correspondientes a los diferentes modelos históricos que lo explican.

Presentan confusión entre los conceptos de sustancia simple y elemento químico. Esta dificultad se presenta fácilmente, ya que para ellos solo existen dos tipos de sistemas materiales: mezclados y no mezclados.

La mayoría de los estudiantes al no disponer del concepto macroscópico de sustancia opuesto al de mezcla, piensa que "todos los materiales que se nos presentan son mezclas (o sustancias), formadas por otras sustancias puras más simples", que suponemos serán los elementos químicos. Sería en cierto modo, un modelo mental o una forma de pensar similar al paradigma aristotélico en cuanto a que se simplifica la complejidad de la composición de los materiales existentes al manifestar explícitamente que todos son mezclas. Se comprende así que se utilice el término "sustancia pura" ya que se presupone que existen, por oposición, “sustancias impuras” (Furió-Más \& Domínguez-Sales, 2007, p. 250).

No saben distinguir entre los conceptos de mezcla y compuesto en los dos niveles de representación. Esta diferenciación se ve afectada porque los estudiantes creen que en el compuesto ya existen las sustancias simples que lo forman. Estos resultados también fueron encontrados por Furió-Más y Domínguez-Sales (2007).

\section{Modelos históricos del elemento químico}

En la investigación se ha demostrado que cuando no hay un aprendizaje significativo sobre algún concepto científico, es necesario revisar su enseñanza. Se cree que una de las tantas razones de esta falta de aprendizaje en los estudiantes puede deberse al desconocimiento de los profesores sobre la historia de la ciencia -desarrollo de los modelos históricos hasta los actuales-, así como a la existencia de visiones epistemológicas sobre la naturaleza de la ciencia y la actividad científica (Fernández et al., 2002; Furió-Más, 1994; McComas et 
al., 1998). La transmisión de estas visiones deformadas, específicamente en la enseñanza de la química, se manifiesta muchas veces de manera implícita en la organización y secuenciación de los contenidos de los libros de texto y en el profesorado, lo cual permea la enseñanza del CEQ².

Por otro lado, es importante que el profesorado cuente con conocimientos sobre la historia de la química, con el fin de utilizarla como una herramienta para definir los conceptos estructurantes - en este caso, el CEQ - y también como tema de clase en donde se pueden analizar o indicar las dificultades que hubo para desarrollarlos, y los problemas conceptuales y obstáculos que fue necesario superar (López-Valentín, 2013).

A continuación, se muestra una síntesis de los modelos históricos en los que se ha introducido el CEQ para explicar los cambios químicos (López-Valentín, 2008; LópezValentín \& Furió-Más, 2020; López-Valentín \& Furió-Más, 2021).

\section{Tabla 1}

Sintesis de los modelos históricos en los que se ha introducido el concepto de elemento químico para explicar los cambios químicos

\begin{tabular}{|c|c|c|c|c|}
\hline Modelo & Aristotélico escolástico & $\begin{array}{c}\text { Macroscópico } \\
\text {-empirista- } \\
\text { Modelo macro }\end{array}$ & $\begin{array}{l}\text { Atómico clásico } \\
\text { Modelo micro I }\end{array}$ & $\begin{array}{l}\text { Submicroscópico } \\
\text { mecánico cuántico } \\
\text { Modelo micro II }\end{array}$ \\
\hline $\begin{array}{l}\text { Científico } \\
\text { precursor }\end{array}$ & Aristóteles & Lavoisier y otros & Dalton y otros & Moseley y otros \\
\hline $\begin{array}{l}\text { Definición } \\
\text { de } \\
\text { elemento }\end{array}$ & $\begin{array}{l}\text { Una de las } 4 \text { formas - } \\
\text { tierra, agua, aire y fuego-- } \\
\text { y } 3 \text { principios - mercurio, } \\
\text { azufre y sal_- metafísicos } \\
\text { que imprimen determinadas } \\
\text { cualidades a la materia } \\
\text { prima². } \\
\text { Cualquier cuerpo real es } \\
\text { una mezcla de elementos } \\
\text { en diferente proporción. } \\
\text { Estos elementos explican } \\
\text { cualitativamente las } \\
\text { propiedades macroscópicas } \\
\text { de los cuerpos. } \\
\text { A cada elemento se le } \\
\text { asocia una propiedad o } \\
\text { dos en particular. Por ello, } \\
\text { el énfasis en una nueva } \\
\text { propiedad obliga a la } \\
\text { introducción de nuevos } \\
\text { "principios" o elementos. }\end{array}$ & $\begin{array}{l}\text { Se introduce el } \\
\text { concepto de sustancia } \\
\text { como aquella que no } \\
\text { es susceptible de ser } \\
\text { descompuesta mediante } \\
\text { manipulaciones } \\
\text { en el laboratorio } \\
\text { —definición } \\
\text { operacional—y } \\
\text { también el de sustancia } \\
\text { compuesta como } \\
\text { aquella que sí se puede } \\
\text { descomponer. }\end{array}$ & $\begin{array}{l}\text { Los átomos } \\
\text { de un mismo } \\
\text { elemento se } \\
\text { caracterizan } \\
\text { por poseer } \\
\text { propiedades } \\
\text { extensivas como } \\
\text { masa y volumen } \\
\text { específicos. }\end{array}$ & $\begin{array}{l}\text { Sistema material } \\
\text { formado por } \\
\text { un conjunto de } \\
\text { átomos y/o iones } \\
\text { elementales que } \\
\text { tienen el mismo } \\
\text { número atómico, lo } \\
\text { que significa tener } \\
\text { la misma carga } \\
\text { nuclear }\end{array}$ \\
\hline
\end{tabular}

1 Si el lector desea conocer las implicaciones en la enseñanza de estas visiones deformadas, puede consultar el manuscrito sobre el actual CEQ (López-Valentín \& Furió-Más, 2021).

2 Cabe aclarar que los tres principios metafísicos no eran conocidos por Aristóteles. Fueron añadidos al modelo mucho tiempo después. 
Modelo de aprendizaje como investigación orientada para la secuenciación de las actividades

De acuerdo con Méheut (2004), una secuencia didáctica o de enseñanza-aprendizaje se entiende como el diseño de una serie de actividades orientadas al aprendizaje de un tema específico. Esta secuencia tiene un carácter dual, pues involucra actividades de investigación y el desarrollo de objetivos relacionados directamente con la enseñanza y el aprendizaje de un tema en particular. Este tipo de secuencias está vinculado con la investigaciónacción. Se decidió optar por una propuesta de orientación socioconstructivista como es el modelo de enseñanza-aprendizaje como actividad de investigación orientada (Furió-Más \& Furió-Gómez, 2009; Guisasola et al., 2008). El modelo se basa en el tratamiento específico de situaciones problemáticas abiertas, lo que fomenta que los estudiantes discutan en pequeños grupos sobre actividades bajo la supervisión del profesor, se familiaricen con los procedimientos científicos, argumenten soluciones y puedan desarrollar sus habilidades cognitivas, metacognitivas y sociales (López-Valentín \& Furió-Más, 2013, p. 1999). En este modelo se pone énfasis en las siguientes estrategias didácticas (Furió-Más \& Furió-Gómez, 2009):

- Debatir y valorar el interés personal y social que tiene conocer la temática a estudiar

-relaciones Ciencia-Tecnología-Sociedad-Ambiente [CTSA]—.

- Analizar cualitativamente las situaciones problemáticas.

- Emitir hipótesis sobre posibles soluciones. Los estudiantes tendrán oportunidad para usar funcionalmente sus concepciones en las predicciones que hagan.

- Elaborar estrategias de resolución o diseñar experimentos para cuestionar las hipótesis.

- Relacionar las soluciones con problemas cotidianos, es decir, seguir enfatizando la inclusión de relaciones CTSA.

\section{Secuencia didáctica que permite introducir la idea actual del CEQ en $3^{\circ}$ de la ESO}

La introducción del CEQ en el modelo atómico daltoniano - que es en el que se va a poner énfasis en la ESO - va a ser la solución hipotética a los dos grandes problemas estructurantes de la química:

\section{Figura 1}

Solución hipotética que ofrece la comprensión del elemento químico a los dos grandes problemas estructurantes de la química

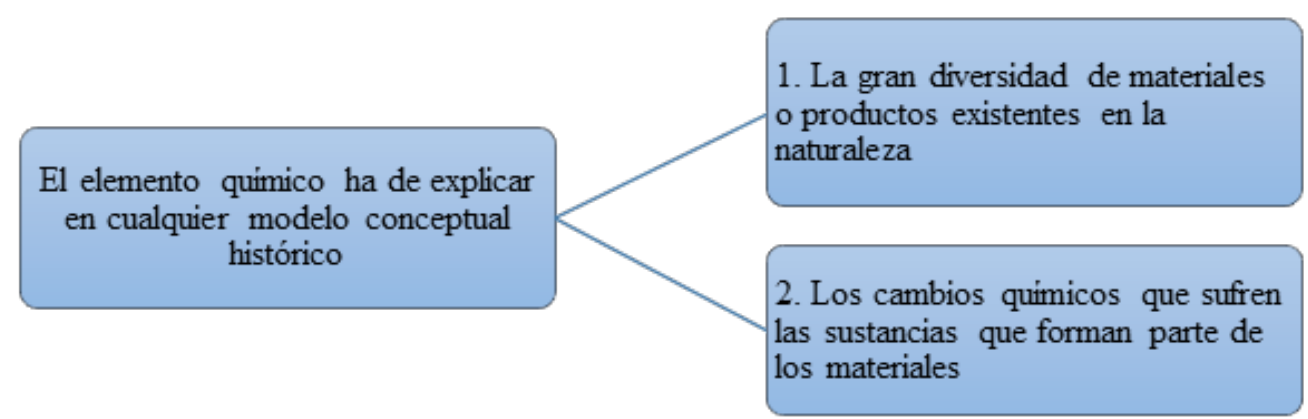


El tratamiento de estos dos problemas requiere que, en principio, se aborden los conceptos de sustancia - simple y compuesta-y de reacción química, a nivel macroscópico, como cambio sustancial. Se necesita que, paulatinamente, vayan interpretándose, a nivel microscópico, con la introducción del CEQ definido en el marco atomista y su conservación en los procesos químicos.

Como hilo conductor, se ha partido del supuesto de que el estudio de estos dos problemas se puede secuenciar y organizar en dos unidades didácticas: una dedicada a la diversidad de materiales; y otra, a la de sus transformaciones químicas. En este trabajo solo nos dedicaremos a la primera parte, es decir, a la diversidad de materiales. Respecto a la segunda parte, solo hemos introducido superficialmente la idea de cambio químico como cambio sustancial y el segundo postulado de la teoría atómica molecular de Dalton. Para llevar a cabo el planteamiento del programa de actividades en cuestión, se tuvieron en cuenta los temas o contenidos que los alumnos han estudiado anteriormente - currículo escolarpara evitar repetirlos. También se dedicó el tiempo necesario a una revisión general del currículo, con el fin de asegurar una correcta comprensión de los nuevos contenidos.

Se diseñó una secuencia de contenidos cuya estructura en forma de problemas/cuestiones se resume en la tabla 2, la cual se compone de tres columnas: la primera muestra la secuencia de situaciones problemáticas que sirve de hilo conductor de la secuencia didáctica; la segunda se centra en los conceptos, procedimientos y actitudes a conocer respecto a la ciencia; y la tercera expone las explicaciones científicas que deben ser aprendidas.

La secuencia didáctica que se presenta a continuación comparte las primeras actividades de una investigación previa (Furió-Más et al., 2012), puesto que tienen en común el punto de partida. En la investigación mencionada se plantea el diseño e implementación de una secuencia de enseñanza para introducir los conceptos de sustancia y compuesto químico. Por eso, no se expondrán en este manuscrito, en donde se empezará a partir de las sustancias simples y compuestas, cuya diferenciación se basa en procesos químicos de composición y descomposición, que son los que se relacionan con la comprensión del CEQ.

Tabla 2

Secuencia didáctica para la enseñanza de "La diversidad y unidad de estructura de los materiales"

\begin{tabular}{cc}
\hline $\begin{array}{c}\text { Secuencia de } \\
\text { situaciones }\end{array}$ & $\begin{array}{c}\text { Conceptos, procedimientos y } \\
\text { actitudes a conocer respecto }\end{array}$ \\
problemáticas & E la ciencia \\
\hline
\end{tabular}

$\begin{array}{ll} & \begin{array}{l}\text { Plantear la emisión de } \\ \text { hipótesis, fundamentadas } \\ \text { en los conocimientos }\end{array} \\ \text { ¿Puede una } & \begin{array}{l}\text { disponibles, susceptibles de } \\ \text { sustancia }\end{array} \\ \text { orientar el tratamiento de las } & \text { situaciones. } \\ \text { otras más sencillas? } & \text { Plantear la elaboración de } \\ & \text { estrategias incluyendo los } \\ & \text { diseños experimentales. }\end{array}$




\begin{tabular}{ll}
\hline & $\begin{array}{l}\text { Plantear la emisión de } \\
\text { hipótesis, fundamentadas en }\end{array}$ \\
¿Cómo se puede & los conocimientos disponibles, \\
distinguir entre & susceptibles de orientar el \\
una mezcla y & tratamiento de las situaciones \\
una sustancia & y de hacer explícitas las ideas \\
compuesta? & previas a las que se prestará \\
& especial atención. \\
\hline
\end{tabular}

Se requerirá introducir la idea de elemento químico para explicar la diversidad de materiales que nos rodean.
¿Cómo podemos explicar de manera unitaria la gran diversidad de materiales que conocemos?
A partir de la organización de las experiencias, la emisión de estrategias adecuadas, elaborar un modelo explicativo inicial. hipótesis y la selección de las

Diferenciación entre mezcla y compuesto.

Se definirá elemento químico como conjunto de átomos de la misma masa con los que se pueden formar toda una serie de sustancias simples - elementales, es decir, formadas por un único elemento-y compuestas —formadas por dos o más elementos-.

Se empezará aquí a aplicar aquellos postulados de Dalton que hacen referencia a la composición de la gran diversidad de sustancias que hay con la combinación de unos pocos elementos.

Se introducirá el concepto microscópico de sustancia - formada por partículas iguales, bien sean átomos o moléculas - cuyas propiedades van a depender de su composición atómica, haciendo énfasis en que se diferencia de una mezcla también a nivel atómico.

¿Cómo se representan las mezclas y las Familiarizarse con sustancias simples observaciones empíricas y el y compuestas desde uso de modelos en la ciencia un punto de vista microscópico?
Al mismo tiempo se definirá a la sustancia simple o elemental como aquella que solo contiene un elemento químico y a la sustancia compuesta como aquella que está formada por varios elementos químicos. Resaltar la idea de que los elementos se caracterizan por tener átomos iguales, de la misma naturaleza y masa, pero sin que conformen estructura alguna uniones de átomos en moléculas-. Por lo tanto, el mismo elemento puede dar lugar a varias sustancias elementales o simples y compuestas

Se ha de tener en cuenta que el alumnado ya maneja una idea de elemento en el lenguaje cotidiano que convendrá debatir para presentar el problema de que no se puede confundir elemento químico con sustancia simple. Por ejemplo, aludir al caso cotidiano "el calcio de la leche" —o el del agua potable dura — o "el hierro de la sangre", es obvio que no se pueden confundir con las sustancias simples calcio y hierro, puesto que tienen diferentes propiedades macroscópicas, pero se ha de advertir que el elemento Ca es el mismo en todas las sustancias o mezclas de sustancias que tengan $\mathrm{Ca}$ —en el modelo daltoniano serán siempre átomos de Ca con la misma masa atómica-. 


\author{
¿Podemos entender \\ ahora lo que \\ significa diversidad \\ y unidad de \\ estructura de los \\ materiales?
}

Introducir la simbología de manera que a cada tipo de átomo se le asocie un elemento que puede dar lugar a moléculas con el mismo tipo de átomos o con distintos tipos de átomos. Se puede hacer ver cómo con los elementos se puede multiplicar el número de sustancias simples y compuestas, y así explicar la gran diversidad de sustancias existentes.

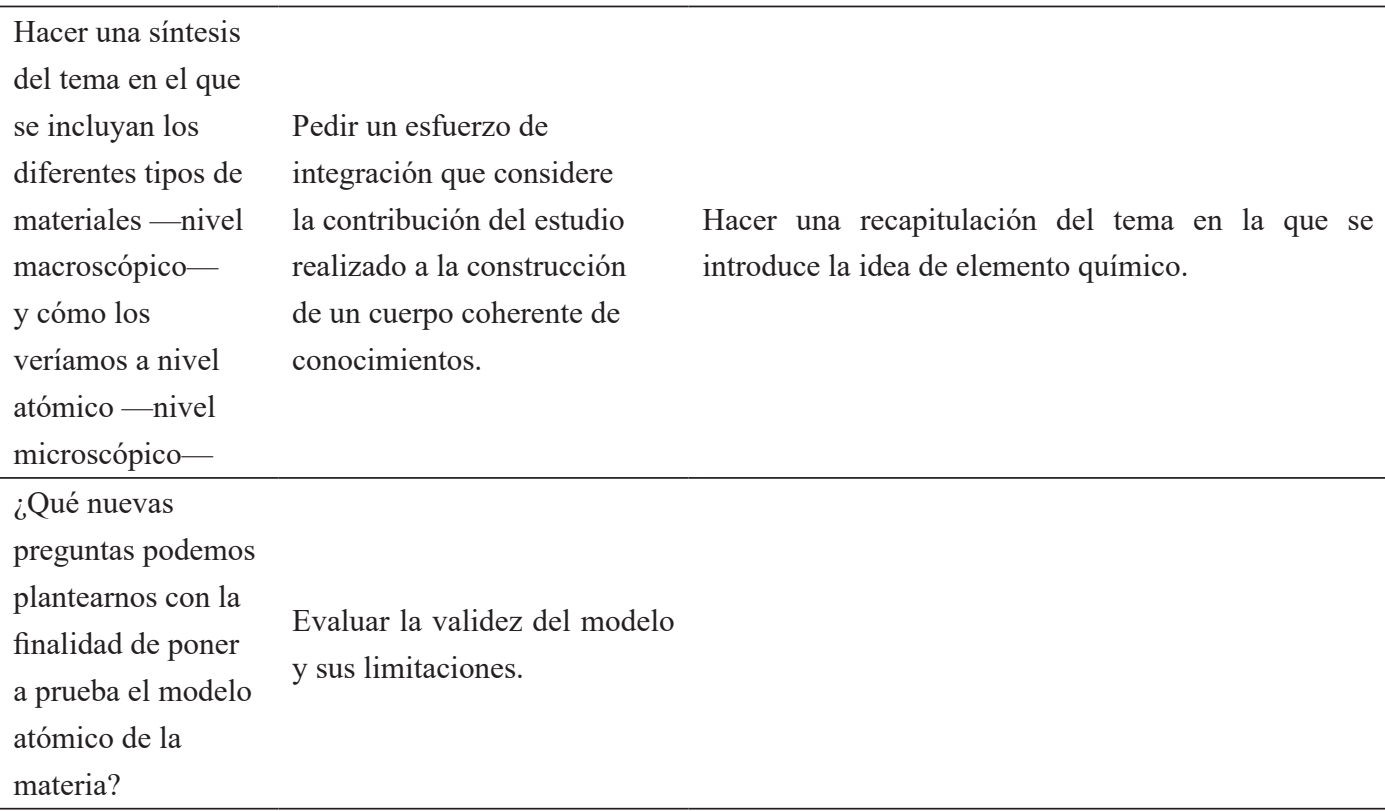

\section{Implementación y validación de la secuencia didáctica}

El trabajo en clase se organizó en pequeños grupos de trabajo en donde los estudiantes discutían sobre las actividades, evaluaban sus ideas iniciales y generaban nuevas ideas para finalmente establecer o no acuerdos.

La figura tradicional del profesor cambia, pues este no practica una clase expositiva, sino que interactúa con los estudiantes mientras ellos discuten. Él guía las discusiones sin dar la respuesta correcta, para que los estudiantes no se disipen. Al finalizar las actividades, un representante de cada grupo comparte sus respuestas al resto de la clase, situación que genera un debate entre los diferentes grupos. De la discusión se obtienen conclusiones consensuadas para reformular las respuestas.

La secuencia didáctica fue diseñada por la autora de este trabajo, dirigida y aplicada al grupo experimental en un curso de $3^{\circ}$ de la ESO en Valencia capital. Participaron treinta estudiantes con una edad promedio entre 14 y 15 años. Para el grupo control, se pidió apoyo a una profesora del mismo instituto. Ella tenía 10 años de experiencia como docente. La profesora trabajaría con sus estudiantes de manera habitual el tema de Diversidad y unidad de estructura de la materia y al final aplicaría el mismo instrumento que el grupo experimental (anexo 1). En total participaron 68 estudiantes en el grupo control.

Para validar la secuencia didáctica, se elaboró un cuestionario (anexo 1). Cada uno de los ítems tiene un propósito y un criterio de valoración. Cada ítem fue validado por dos investigadores expertos. El análisis de las respuestas a los ítems del cuestionario fue 
realizado por separado también por dos investigadores - incluyendo la autora- $-\mathrm{y}$, en caso de existir discrepancias en alguno, este se revisaba nuevamente. Si subsistían, se eliminaban o se solicitaba la intervención de un tercero. Para clasificar las respuestas como correctas o incorrectas, no se tuvo en cuenta únicamente el resultado, sino también la explicación y la argumentación utilizadas.

Para determinar si existían diferencias significativas entre el grupo experimental y los grupos control, cada ítem fue comparado mediante el parámetro estadístico no paramétrico chi cuadrado $\left(X^{2}\right)$. Se considera que la asociación entre el método de enseñanza y los resultados de aprendizaje obtenidos es significativa si el parámetro estadístico $X^{2}$ obtenido con el $95 \%$ de confianza tiene un valor mayor que el valor teórico correspondiente (Crocker, 1969; Heumann \& Schomaker, 2016).

\section{Resultados y análisis de resultados}

Se analizarán comparativamente las respuestas al cuestionario (anexo 1) obtenidas por los grupos experimentales - intervención didáctica dirigida por la autora-y por los grupos control -enseñanza habitual con su profesora-. En la tabla 3 y en la gráfica 1, se muestran los porcentajes de las respuestas correctas. El valor de $X^{2}$ se obtiene de la comparación entre el promedio de los grupos experimentales y los de control. Su valor es más alto que el proporcionado por las tablas de contingencia para todos los ítems. Por lo tanto, la hipótesis nula debe ser rechazada en su máxima significación de 1 por cada 1000, una puntuación considerablemente inferior al 5\%, que es normalmente el máximo aceptado para este tipo de investigaciones (Furió-Más et al., 2012, p. 119).

Tabla 3

Resultados obtenidos en el cuestionario por los grupos experimentales y de control

\begin{tabular}{|c|c|c|c|c|}
\hline \multirow[b]{2}{*}{ Ítem } & \multirow[b]{2}{*}{ Objetivo del ítem } & \multicolumn{2}{|c|}{ Porcentaje de respuestas correctas (\%) } & \multirow[b]{2}{*}{$X^{2}$} \\
\hline & & $\begin{array}{l}\text { Grupo experimental } \\
\qquad(\mathrm{N}=\mathbf{3 0})\end{array}$ & $\begin{array}{l}\text { Grupo control } \\
\qquad(N=68)\end{array}$ & \\
\hline 1 & $\begin{array}{l}\text { Diferenciar procedimentalmente una mezcla } \\
\text { de sustancias - material— de una sustancia. }\end{array}$ & 36,6 & 4,4 & 19,25 \\
\hline 2 & $\begin{array}{l}\text { Reconocer la conceptualización macroscópica } \\
\text { de la sustancia química. }\end{array}$ & 73,3 & 30,8 & 15,27 \\
\hline 3 & Definir el concepto de elemento químico. & 46,6 & 7,3 & 25,41 \\
\hline 4 & $\begin{array}{l}\text { Distinguir entre elemento químico y sustancia } \\
\text { simple. }\end{array}$ & 63,3 & 10,2 & 29,22 \\
\hline 5 & $\begin{array}{l}\text { Distinguir el total de elementos químicos } \\
\text { presentes en un conjunto de sustancias. }\end{array}$ & 76,6 & 13,2 & 38,42 \\
\hline
\end{tabular}

Como podemos notar en la tabla 3 y en la gráfica 1, las diferencias entre los grupos experimentales y los de control son estadísticamente significativas para todos los ítems. A continuación, se expondrán los resultados correspondientes a los seis ítems.

Respecto al ítem 1, cuyo objetivo era saber si los estudiantes diferencian procedimentalmente una mezcla de sustancias de una sustancia, el criterio de valoración 
era que el estudiante fuese capaz de describir que las sustancias tienen ciertas propiedades específicas que son constantes e invariables. En cambio, las propiedades de una mezcla dependen de su composición, que es variable. Por ejemplo, la parafina funde en un rango de 40 a $65^{\circ} \mathrm{C}$, ya que es una mezcla de hidrocarburos cuyos puntos de fusión se encuentran en ese intervalo.

\section{Gráfica 1}

Representación gráfica - porcentaje- de los resultados obtenidos en el cuestionario por los grupos experimentales $y$ de control

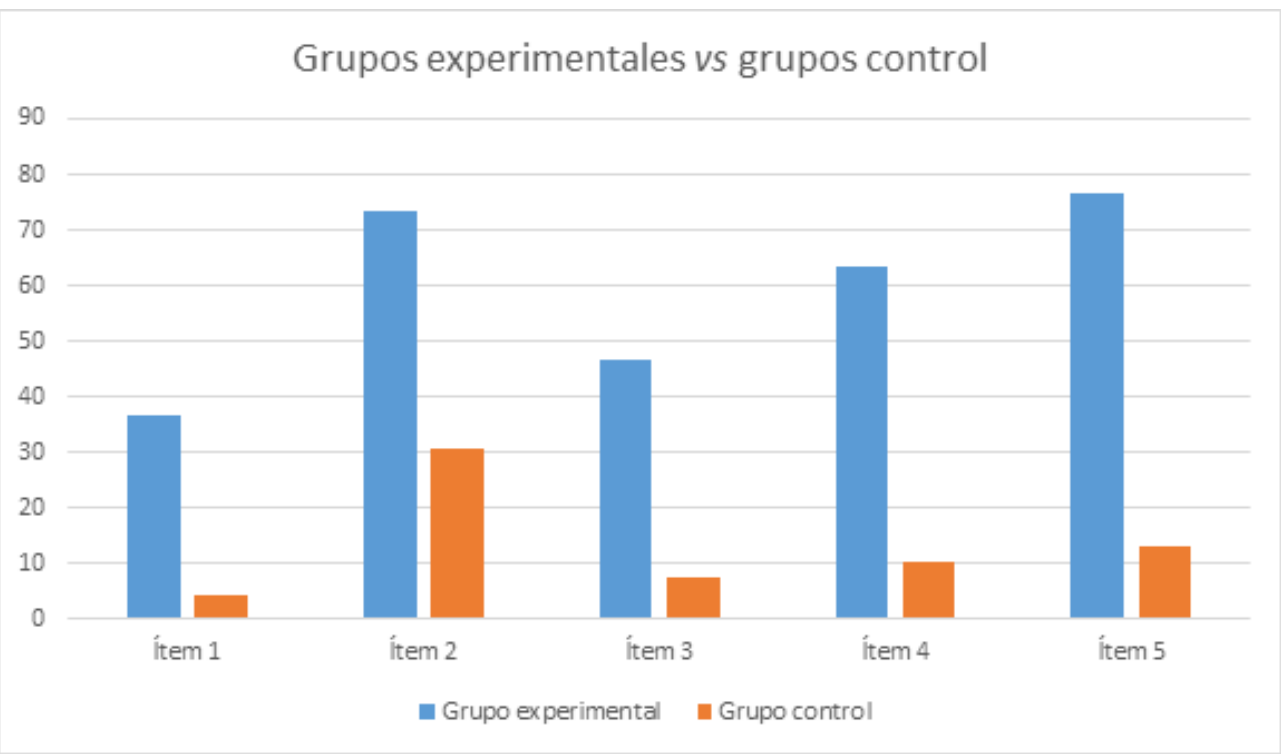

Los resultados obtenidos nos permiten afirmar que los estudiantes de los grupos experimentales han logrado una mayor diferenciación entre una mezcla y una sustancia $-36,6 \%$ - que la expresada por los estudiantes de los grupos control - 4,4\%-. Un ejemplo de una respuesta correcta es el siguiente:

- ESO_20: En una mezcla se podrían separar los componentes mediante operaciones

físicas como filtración o decantación. En cambio, una sustancia hay que descomponerla mediante operaciones químicas como la electrólisis.

En cuanto a las respuestas incorrectas, se encontró que este porcentaje disminuye un 23,4 \% en los estudiantes de los grupos experimentales - 63,3\% - respecto a los estudiantes de los grupos control -86,7 \%-, quienes no saben cómo diferenciar experimentalmente una mezcla de una sustancia. Estos resultados son muy similares a los reportados por FurióMás y Domínguez-Sales (2007), en donde encontraron que la mayoría de los estudiantes no se han apropiado de la definición operacional de sustancia química en el nivel macroscópico y asocian el significado de este término con el más genérico de material o producto -71,4\%-, que habitualmente utilizamos para nombrar las mezclas.

En relación con el ítem 2, cuyo objetivo era que el estudiante reconociera la conceptualización macroscópica de la sustancia química, el criterio de valoración de las respuestas evidenciaba que el estudiante fuese capaz de reconocer y justificar la elección de, al menos, dos propiedades características cuantitativas - por ejemplo, temperatura de 
fusión, temperatura de ebullición y/o densidad- que permiten la caracterización de una sustancia, aunque se añadieran las propiedades características cualitativas —color, olor, etc.-, que son poco diferenciadoras.

Como indican los resultados, los estudiantes de los grupos experimentales logran un porcentaje de respuestas correctas mayor - 73,3\%- respecto a los grupos control $-30,8 \%$ - al reconocer y justificar la elección de, al menos, dos propiedades características cuantitativas que permiten la conceptualización macroscópica de sustancia, sin dejar de lado las propiedades cualitativas. Un ejemplo prototípico es la siguiente respuesta:

- ESO_9: Porque cada sustancia tiene unas características propias que solo le pertenecen a ella. Por el olor se podría saber en algunos casos, pero es más impreciso que las características propias que son: densidad, temperatura de ebullición y temperatura de fusión.

En este caso, el alumno reconoce que el olor podría ayudarle a distinguir una sustancia, aunque sabe que no es método exacto ni correcto - podría enfrentarse a una mezcla de sustancias inodora-, por lo que prefiere la elección de las propiedades características de la sustancia en cuestión.

$\mathrm{Al}$ analizar en conjunto los resultados de los dos ítems anteriores, podemos resumir que los estudiantes de los grupos experimentales presentan un porcentaje mayor - 73,3\%de respuestas correctas con respecto a los grupos control -30,8\%- en la definición procedimental de sustancia química en el nivel macroscópico. Son capaces de distinguir entre los conceptos de sustancia y material, concebido como mezcla de sustancias - 36,6\% y 4,4\%, respectivamente- Sin embargo, estos últimos resultados no son del todo satisfactorios, pues el 36,6 \% obtenido por los grupos experimentales implica que -como ya se indica en el trabajo de Furió-Más y Domínguez-Sales (2007) y en el de Furió et al., (2012) - este concepto empírico de sustancia es bastante difícil de adquirir.

El turno ahora es para el ítem 3, cuyo objetivo era conocer la definición de elemento químico que tienen los estudiantes. El criterio de valoración para este ítem fue que el estudiante definiera el elemento químico con base en el modelo daltoniano - conjunto de átomos iguales en masa y/o volumen-.

Como se puede observar, los estudiantes de los grupos experimentales definen correctamente el concepto de elemento químico en el marco de la teoría daltoniana con un porcentaje del $46,6 \%$, a diferencia de los grupos control, quienes solo alcanzan un porcentaje del 7,3\%. Como ejemplo de respuesta correcta, está la siguiente afirmación:

- ESO_15: Un elemento químico es aquel [que] no tiene estructura molecular característica porque está formado por átomos del mismo elemento. Es, por ejemplo, el hidrógeno $(\mathrm{H})$, porque si lo viéramos microscópicamente solo veríamos átomos del mismo tipo, todos iguales.

Este estudiante afirma que el elemento químico carece de estructura molecular, pues es una construcción elaborada para entender y clasificar la diversidad. En la naturaleza no existen las sustancias - simples y compuestas-. Diferencia el CEQ implícitamente del concepto de sustancia simple. También, de manera explícita, presenta su definición según el modelo daltoniano. Habrá que tener cuidado e indagar mejor, en futuras ocasiones, a qué se 
refiere con el término microscópico cuando habla de los átomos, puesto que puede inducir al alumnado a pensar que los átomos pueden "verse" en el microscopio. Lo único que le faltó decir es que la característica común en todos los átomos es su masa.

El objetivo del ítem 4 era que el estudiante definiera el elemento carbono como un conjunto de átomos con el mismo número atómico o la misma masa atómica y, en consecuencia, distinguiera entre elemento químico y sustancia simple. En este caso concreto, el estudiante ha de saber que el elemento carbono puede estar presente en las sustancias simples -como el grafito y el diamante- y en compuestos - como el dióxido de carbono-. Por lo tanto, el criterio de valoración para este ítem era que el estudiante fuese capaz de indicar que las dos sustancias simples mencionadas - el grafito y el diamante - son diferentes, pero están conformadas por el mismo elemento: carbono.

Como puede observarse en los resultados obtenidos, los estudiantes experimentales obtuvieron un 63,3\% de respuestas correctas frente a los estudiantes control, que alcanzaron solamente un 10,2\%. A continuación, mostramos una respuesta que cumplió con el objetivo planteado:

- ESO_1: Es un elemento químico (C), que, viéndolo microscópicamente, según la sustancia [de la] que forma parte, sus átomos pueden estar organizados de diferente manera. En el grafito estarán más separados y las fuerzas de unión serán más débiles, por eso es más frágil que el diamante. En este último están colocados de otra forma y las fuerzas de unión son más fuertes, por eso es más resistente.

Aunque el estudiante no definió explícitamente el elemento carbono según la teoría daltoniana, ha expresado claramente que el elemento carbono puede formar varias sustancias. Aunque el estudiante indica que estas sustancias sean las indicadas en la respuesta, podemos interpretar que, en el peor de los casos, se refiere a ellas y, por lo tanto, distingue entre el elemento carbono y las sustancias simples.

Respecto a los resultados del ítem 5, cuyo objetivo era saber si los estudiantes distinguían el total de elementos químicos presentes en las sustancias anteriores, se trata de ver si se presenta la dificultad de aprendizaje según la cual se considera que el elemento químico es la sustancia simple. En este último caso, es de esperar que digan que solamente hay dos elementos químicos - el oxígeno y el cloro-. El criterio de valoración positivo será si el estudiante identifica que en las sustancias mencionadas están presentes cinco elementos $-\mathrm{O}, \mathrm{Na}, \mathrm{H}, \mathrm{C}$ y $\mathrm{Cl}-$.

Como podemos observar, el 76,6 \% de los estudiantes de los grupos experimentales dan una respuesta correcta frente a los estudiantes de los grupos de control, quienes solo presentaron un porcentaje positivo del 13,2 \%. Respecto a las respuestas erróneas, los estudiantes experimentales solo se equivocaron en un 16,6 \% y los estudiantes del grupo control en un 70,5\%. Dentro de las respuestas equivocadas de los grupos de control, solo un estudiante de los treinta mencionó que había dos elementos químicos -el oxígeno $\left(\mathrm{O}_{2}\right)$ y el cloro $\left(\mathrm{Cl}_{2}\right)$ - Identificó explícitamente el elemento químico con la sustancia simple. En cambio 13 de los 68 estudiantes presentaron explícitamente esta confusión -19,1\%—.

Como resumen, se señalan los resultados obtenidos en estos dos ítems -4 y $5-$. Los estudiantes experimentales diferencian mejor los conceptos de sustancia simple y elemento 
químico con un porcentaje promedio del $70 \%$ respecto a los estudiantes de los grupos control $-11,7 \%$ -

Por último, en el ítem 6, cuyo objetivo era saber si los estudiantes diferenciaban los conceptos de mezcla, sustancias simples y sustancias compuestas a nivel microscópico, la respuesta es correcta si identifican las muestras de la siguiente manera:

Tabla 4

Respuestas correctas para el ítem 6

\begin{tabular}{lccl}
\hline \multicolumn{1}{c}{$\mathbf{1}$} & $\mathbf{2}$ & $\mathbf{3}$ & \multicolumn{1}{c}{$\mathbf{4}$} \\
\hline $\begin{array}{l}\text { Mezcla de 2 sustancias } \\
\text { simples }\end{array}$ & 1 sustancia compuesta & 1 sustancia simple & $\begin{array}{l}\text { Mezcla de 2 sustancias } \\
\text { compuestas }\end{array}$ \\
\hline
\end{tabular}

Por lo que respecta a la definición de sustancia, se considerará como correcta si la definen microscópicamente como un conjunto de partículas iguales -átomos o moléculas-, que tienen una composición y estructura definidas; o bien, macroscópicamente, como material puro que tiene un conjunto de propiedades características invariables, como temperaturas de ebullición y de fusión, densidad, índice de refracción, conductividades eléctrica y calorífica, calor específico, etc.

Tabla 5

Resultados del item 6, respecto a la diferenciación microscópica de los conceptos mezcla, sustancia simple y sustancia compuesta

\begin{tabular}{|c|c|c|c|c|}
\hline Ítem & Objetivo del ítem & \multicolumn{2}{|c|}{$\begin{array}{c}\text { Porcentaje de respuestas } \\
\text { correctas }(\%)\end{array}$} & \multirow[b]{3}{*}{$X^{2}$} \\
\hline 6 & $\begin{array}{l}\text { Saber si diferencian los conceptos de mezcla, sustancias } \\
\text { simples y sustancias compuestas a nivel microscópico }\end{array}$ & \multirow{2}{*}{$\begin{array}{c}\text { Grupo } \\
\text { experimental } \\
(\mathbf{N}=\mathbf{3 0})\end{array}$} & \multirow{2}{*}{$\begin{array}{l}\text { Grupo } \\
\text { control } \\
(\mathrm{N}=68)\end{array}$} & \\
\hline Cuadro & Descripción del cuadro & & & \\
\hline 1 & Mezcla de 2 sustancias simples (M2SS) & 50 & 10,2 & 20,12 \\
\hline 2 & 1 sustancia compuesta (1SC) & 70 & 64,7 & 3,42 \\
\hline 3 & 1 sustancia simple (1SS) & 96,6 & 76,4 & 6,32 \\
\hline \multirow[t]{2}{*}{4} & Mezcla de 2 sustancias compuestas (M2SC) & 56,6 & 5,8 & 17,28 \\
\hline & Definición de sustancia & 60 & 1,4 & 32,43 \\
\hline
\end{tabular}

En este ítem 6 se analizará si los estudiantes saben distinguir entre los conceptos de mezcla y compuesto en el nivel microscópico de representación. Por otro lado, se busca saber si definen correctamente el concepto de sustancia desde el punto de vista microscópico o macroscópico. Esta competencia es importante para establecer una relación adecuada entre los conceptos de sustancia - especialmente el de sustancia simple- y el de elemento químico. Los resultados obtenidos se analizarán por separado, es decir, se revisarán las respuestas de los cuatro cuadros pertenecientes a este ítem (tabla 5). Al final se analizarán y se compararán junto con la definición general de sustancia que dan los estudiantes de ambas muestras:

\section{Cuadro 1}

Como podemos ver, los estudiantes de los grupos experimentales reconocen una 
mezcla de dos sustancias simples, al obtener un porcentaje del $50 \%$, mientras que la muestra de estudiantes control solo logró un porcentaje positivo del 10,2\%. Con respecto a las respuestas incorrectas de los grupos experimentales - 46,6 \%-, la mitad -23,3\%contestó que se trataba de una sustancia compuesta. Este resultado es casi idéntico al porcentaje de estudiantes del grupo control, que contestó lo mismo - $25 \%$-. Se cree que esto puede deberse a que hay solamente dos clases de átomos diferentes y que además están en la misma proporción.

\section{Cuadro 2}

Prácticamente no hay diferencia entre los porcentajes obtenidos - solo un 5,3\%-en ambas muestras de estudiantes, como lo confirma la prueba de distribución de $X^{2}$, en donde el valor de $p$ indica que en el resultado de esta parte del ítem 6 - sustancia compuestaentre las dos muestras de estudiantes no hay diferencias significativas. Podemos esperar obtener un resultado semejante a este en el próximo cuadro 3, en el que se representa una sustancia simple, pues, por simple deducción, los estudiantes podían distinguir fácilmente entre una sustancia simple y una sustancia compuesta al comparar su representación microscópica gráficamente.

\section{Cuadro 3}

Como resultado del análisis del cuadro anterior, comprobamos que no existen diferencias significativas entre las dos muestras de estudiantes, pues, a pesar de que hay una diferencia entre ambas muestras del 20,2\%, la prueba $X^{2}$ nuevamente vuelve a señalar que hay asociación en las muestras y no son independientes. Lo único positivo que podemos señalar en los resultados obtenidos es que la muestra de estudiantes experimentales no presentó alguna respuesta incorrecta y solo hubo una en blanco con respecto a la muestra de estudiantes control.

\section{Cuadro 4}

Los estudiantes de los grupos experimentales reconocen una mezcla de dos sustancias compuestas al obtener un porcentaje del 56,6 \%, mientras que la muestra de estudiantes control solo logró un porcentaje positivo del 5,8 \%. Con respecto al valor de $p(<0,001)$ obtenido, podemos comprobar que sí existen diferencias significativas entre las dos muestras de estudiantes.

Para finalizar el análisis de este ítem 6, revisemos ahora los resultados obtenidos sobre la definición microscópica de sustancia.

El porcentaje de respuestas correctas de los estudiantes experimentales -60\%es mucho mayor que el obtenido por los estudiantes control -1,4\%-. El $60 \%$ de los estudiantes experimentales definió el concepto de sustancia microscópicamente como muestra el siguiente ejemplo:

- ESO_27: Un conjunto de átomos iguales, se clasifican en sustancia compuesta cuando presentan distinto tipo de átomos y en sustancia simple cuando tienen el mismo tipo de átomos.

Los resultados anteriores muestran que, a diferencia de los estudiantes control, los estudiantes experimentales ya empiezan a pensar en átomos y moléculas. Sin embargo, 
como lo expresa el estudiante anterior, podría prestarse a confusión cuando expresa "un conjunto de átomos iguales" y después afirma que pueden ser del mismo tipo de átomos o átomos diferentes. Con respecto al valor de $p(<0,001)$ obtenido, podemos comprobar que sí existen diferencias significativas entre las dos muestras de estudiantes.

Finalmente, podemos corroborar que los estudiantes experimentales muestran una mejor distinción del concepto de mezcla — de sustancias simples o de sustancias compuestas-, ya que presentan porcentajes superiores al $50 \%$ en comparación con los grupos de estudiantes control, así como al definir el concepto microscópico de sustancia -60 \%-.

No obstante, se puede mejorar la enseñanza para llegar a conseguir un porcentaje superior al obtenido por los grupos experimentales.

\section{Conclusiones}

Las conclusiones a este trabajo contestarán las preguntas de investigación formuladas con anterioridad:

¿Cómo diseñar una secuencia didáctica que tenga en cuenta los aspectos mencionados para mejorar el aprendizaje del concepto de elemento químico en estudiantes de secundaria?

Fue posible diseñar una secuencia didáctica para la enseñanza del tema titulado "La diversidad y unidad de estructura de los materiales" para estudiantes de $3^{\circ}$ de la ESO, a partir de las dificultades que presentan los estudiantes respecto a la comprensión del CEQ y su evolución histórica, bajo el modelo de aprendizaje como investigación orientada para secuenciar las actividades propuestas.

¿En qué medida la aplicación de la secuencia didáctica puede favorecer la superación de las dificultades de los estudiantes respecto al CEQ?

Para responder a esta pregunta de investigación, se retomarán las dificultades de los estudiantes reportadas en la literatura especializada y se expondrá en qué medida se superaron esas dificultades tras la aplicación de la secuencia didáctica propuesta:

No entienden el concepto macroscópico de sustancia y lo confunden con el concepto de material -mezcla de sustancias-. Los estudiantes de los grupos experimentales presentan un mejor manejo en la definición procedimental de sustancia química en el nivel macroscópico $-73,3 \%$ - con respecto a los grupos control -30,8 \%-. Asimismo, son capaces de distinguir mejor entre los conceptos de sustancia y material, concebido como mezcla de sustancias $-36,6 \%$-, comparados con los grupos control $-4,4 \%-$. No obstante, como podemos ver, la dificultad en esta diferenciación requiere aún una mayor reiteración en el aprendizaje - en este tema o en los posteriores-.

No comprenden el concepto de elemento químico. Los estudiantes de los grupos experimentales definen mejor el concepto de elemento químico en el marco de la teoría daltoniana con un porcentaje del $46,6 \%$, a diferencia de los grupos control quienes solo alcanzan un porcentaje del 7,3\%.

Presentan confusión entre los conceptos de sustancia pura y elemento químico. Los estudiantes experimentales distinguen mejor los conceptos de sustancia simple y elemento químico con un porcentaje promedio del $70 \%$ respecto a los estudiantes de los grupos control $-11,7 \%$ - Como podemos ver, el porcentaje se ha superado casi seis veces en la 
consecución de este objetivo.

\section{No saben distinguir entre los conceptos de mezcla y compuesto en los dos niveles de representación.}

- Los estudiantes experimentales presentan una mejor distinción del concepto microscópico de mezcla - de sustancias simples o de sustancias compuestas-, ya que presentan porcentajes promedio superiores del $50 \%$, en comparación con los grupos de estudiantes control, con un porcentaje máximo del 10,2\%.

- Los estudiantes experimentales definen mejor el concepto microscópico de sustancia, dado que logran un porcentaje del $60 \%$, en comparación con los estudiantes control, que solo alcanzan el 1,4\%. El resultado obtenido era de esperarse, pues se hizo hincapié en el modelo daltoniano.

Un dato interesante para resaltar es el bajo porcentaje de respuestas en blanco obtenido por los estudiantes de los grupos experimentales — con un máximo de 13,3\% para el ítem 5 del anexo 1, sobre la diferenciación entre sustancia simple y elemento químico-, respecto al obtenido por los estudiantes control, en donde siempre se mantuvo presente para todos los ítems analizados - desde un 8,8 \% para el ítem 1, diferenciación entre material y sustancia, hasta un máximo del $44,1 \%$ para el ítem $4-$. Este resultado puede explicarse debido a que los estudiantes que han seguido un modelo de enseñanza por investigación orientada adquieren una mayor seguridad al contestar una pregunta planteada $y$, por lo tanto, presentan un menor porcentaje de abandono de un problema.

Si bien se lograron considerables porcentajes de avance en los grupos experimentales con respecto a los grupos control, todavía algunos estudiantes muestran problemas al entender el concepto macroscópico de sustancia, que sigue confundiéndose con el concepto de material. Presentan confusión entre los conceptos de sustancia y elemento químico y aún hay poca distinción entre los conceptos de mezcla y compuesto en los dos niveles de representación. Estos resultados sugieren la necesidad de hacer ajustes a las actividades de la secuencia didáctica y de ponerla a prueba en grupos más grandes y con nuevos profesores.

\section{Referencias}

Caamaño, A., Mayós, C., Mestre, G., \& Ventura, T. (1983). Consideraciones sobre algunos errores conceptuales en el aprendizaje de la química en el bachillerato. Enseñanza de las Ciencias, 1(3), 198-200. https://www.raco.cat/index.php/Ensenanza/article/view/50673

Crocker, A. (1969). Statistics for the teacher or how to put figures in their places. Penguin Books.

Fernández, I., Gil, D., Carrascosa, J., Cachapuz, A., \& Praia, J. (2002). Visiones deformadas de la ciencia transmitidas por la enseñanza. Enseñanza de las Ciencias, 20(3), 477-488.

Furió-Más, C. (1994). Tendencias actuales en la formación del profesorado. Enseñanza de las Ciencias, 25(2), 241-258.

Furió-Más, C., \& Domínguez-Sales, C. (2007). Problemas históricos y dificultades de los estudiantes en la conceptualización de sustancia y compuesto químico. Enseñanza de las Ciencias, 25(2), 241-258. https://www.raco.cat/index.php/Ensenanza/article/view/87876

Furió-Más, C., \& Furió-Gómez, C. (2009). ¿Cómo diseñar una secuencia de enseñanza de 
ciencias con una orientación socioconstructivista? Educación Química, (Junio), 246-251.

Furió-Más, C., Domínguez-Sales, C., \& Guisasola, J. (2012). Diseño e implementación de una secuencia de enseñanza para introducir los conceptos de sustancia y compuesto químico. Enseñanza de las Ciencias, 30(1), 113-128. https://dx.doi.org/10.5565/rev/ec/ v30n1.575

Guisasola, J., Furió-Más, C., \& Ceberio, M. (2008). Science Education Based on Developing Guided Research. En M. Thomas (Ed.), Science Education in Focus (pp. 173-202). Nova Publishers, Inc.

Heumann, C. \& Schomaker, M. (2016). Introduction to statistics and data analysis. Springer. https://doi.org/10.1007/978-3-319-46162-5

Laugier, A., \& Dumon, A. (2003). Obstacles épistémologiques et didactiques à la construction du concept d'elément chimique: quelles convergences? Didaskalia, (22), 69-97. https:// doi.org/10.4267/2042/23921

López-Valentín, D. (2008). La enseñanza y el aprendizaje del concepto de elemento químico en la educación secundaria y el bachillerato. Análisis crítico y propuesta de mejora [Tesis doctoral inédita]. Universitat de València, España.

López-Valentín, D. (2013). Presencia de la visión ahistórica y aproblemática de la ciencia en la enseñanza del concepto de elemento químico. En C. Celestino \& M. Brzenzinski (Orgs.). Aprendendo ciencia e sobre sua natureza: abordagens históricas e filosóficas (pp. 451-460). Tipographia Editora Expressa.

López-Valentín, D., \& Furió-Más, C. (2013). Diseño de una secuencia de enseñanza para introducir el concepto de elemento químico en la educación secundaria. Enseñanza de las Ciencias, (Extraordinario), 1997-2001. https://www.raco.cat/index.php/Ensenanza/ article/view/307672

López-Valentín, D., \& Furió-Más, C. (2020). Desarrollo histórico y epistemológico de los conceptos elemento químico, sustancia y sustancia simple (Primera parte). Educación Química, 31(4), 131-143. http://dx.doi.org/10.22201/fq.18708404e.2020.4.75258

López-Valentín, D., \& Furió-Más, C. (2021). El concepto actual de elemento químico: ¿uno o dos significados? Implicaciones en su enseñanza. Segunda parte. Educación Química, $32(1)$.

McComas, W., Clough, M., \& Almazroa, H. (1998). The role and character of the nature of science in science education. En W. McComas (Ed.), The nature of science in education, rationales and strategies (pp. 3-39). Kluwer Academic Publishers.

Méheut, M. (2004). Teaching-learning sequences: aims and tools for science education research. International Journal of Science Education, 26(5), 515-535. https://doi.org/10.1 080/09500690310001614762 


\section{Anexo 1 \\ Cuestionario sobre la diversidad y estructura de los materiales para estudiantes de ciencias de la naturaleza de la ESO}

Nombre: Curso:

Fecha:

1. ¿Cómo podrías saber si un material es una sustancia o una mezcla de sustancias?

2. En el laboratorio se ha perdido la etiqueta de un frasco que contiene un líquido incoloro. Explica cuáles de los siguientes datos te ayudarían a saber de qué sustancia se trata.

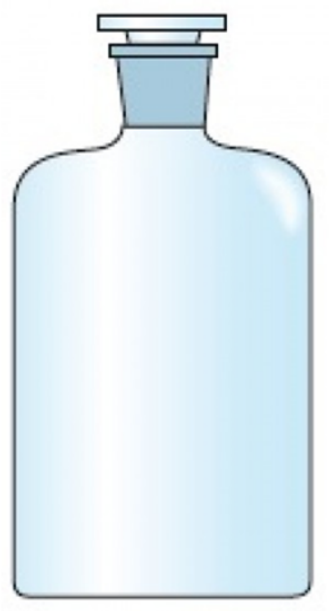

\begin{tabular}{|c|c|}
\hline Masa (g) & 78 \\
\hline Volumen $\left(\mathrm{cm}^{3}\right)$ & 24 \\
\hline $\begin{array}{l}\text { Temperatura del líquido } \\
\left({ }^{\circ} \mathrm{C}\right)\end{array}$ & 20 \\
\hline Temperatura de fusión $\left({ }^{\circ} \mathrm{C}\right)$ & 10 \\
\hline $\begin{array}{l}\text { Temperatura de ebullición } \\
\left({ }^{\circ} \mathrm{C}\right)\end{array}$ & 317 \\
\hline Densidad $\left(\mathrm{g} / \mathrm{cm}^{3}\right)$ & 1.84 \\
\hline Color & incoloro \\
\hline Olor & picante \\
\hline
\end{tabular}

3. ¿Qué es para ti un elemento químico?

4. Se conocen varias sustancias simples que están formadas por el elemento carbono. Las más conocidas son el grafito (que se puede encontrar en la mina de un lápiz) y el diamante. ¿Cómo explicarías lo que es el elemento carbono?

5. Hay materiales ordinarios formados por una sustancia y cuya composición conocemos, como por ejemplo:

a. El oxígeno que hay en el aire $\left(\mathrm{O}_{2}\right)$

b. El bicarbonato de sodio cuya fórmula es $\mathrm{NaHCO}_{3}$

c. El gas butano $\left(\mathrm{C}_{4} \mathrm{H}_{10}\right)$

d. El cloro, $\mathrm{Cl}_{2}$, que hay disuelto en muy pequeñas cantidades en el agua potable

e. La sal común en polvo, que prácticamente es cloruro de sodio $(\mathrm{NaCl})$

f. El dióxido de carbono, $\mathrm{CO}_{2}$, que se desprende de la gaseosa o de los refrescos

Explica cuántos elementos químicos distintos hay en el conjunto de estas seis sustancias: 
6. Clasifica todas las muestras que aparecen en el esquema según sean mezclas, sustancias simples o compuestas. Los átomos diferentes se representan con colores distintos:

En el caso que una de las muestras se considere una mezcla, indica de cuántas sustancias está formada y de qué tipo son (si son simples o compuestas).

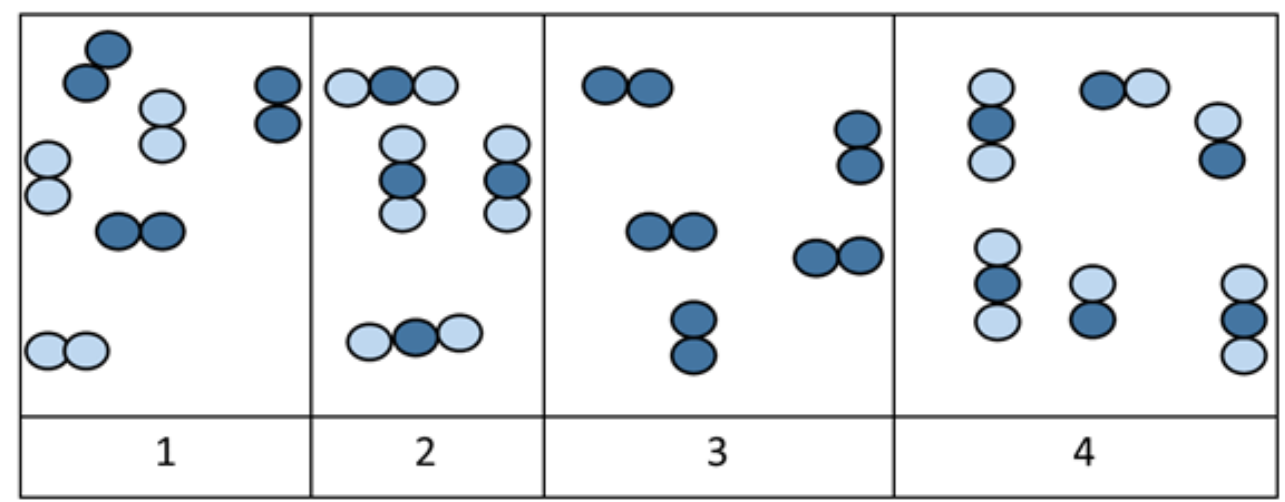

Escribe tus respuestas en cada uno de los recuadros vacíos.

\begin{tabular}{|c|c|c|c|}
\hline & & & \\
& & & \\
\hline 1 & 2 & 3 & 4 \\
\hline
\end{tabular}

Da una explicación de lo que es para ti una sustancia: 